\title{
Design of Fatigue Life Testing Machine for Slewing Bearing Based on LabVIEW
}

\author{
Kang CHEN, Pei-Tao YANG, and Guo-Xiang HOU ${ }^{a}$ \\ School of Naval Architecture \& Ocean Engineering, Huazhong University of Science and \\ Technology, Wuhan, China \\ ahouguoxiang@163.com
}

Keywords: Slow speed slewing bearing, Overturning moment, Fatigue life, Equivalent load, Vibration monitoring.

\begin{abstract}
Slewing bearings generally consist of the rotational connection between two substructures for combined load at very low speeds. Although there are a large number of traditional or modern techniques widely used in slewing bearing, they may not be able to meet the requirements of bearing industry precisely due to the huge difference between the general bearing and the slewing bearing, thus, the experiments are the most effective and reliable methods. In this paper a special test machine for slewing bearing is presented and an accelerating fatigue life test method based on the test equipment is proposed to predict the whole fatigue life of the slewing bearing. A virtual instrument test system using vibration detection whose hardware was constructed by signal conditioning, data acquisition card and the industrial personal computer was built. Its software platform was based on LabVIEW. Then the hardware configuration, the sensor mounting arrangement and the use of structured and modular approaches to software programming are described. It is shown that the presented test equipment can meet the high reliability requirements of the fatigue life test for slewing bearing under the actual working conditions.
\end{abstract}

\section{Introduction}

Slewing bearing is a kind of large-sized special rolling bearing, it is widely used in ship machinery, aerospace, lifting machinery, construction machinery, wind power and other fields. Considering its special structure which can sustain axial load, radial load and overturning torque, the current calculation methods of equivalent load and service life which have been widely used cannot be directly applied to the design and model selection of slewing bearing because of this kind of mechanical loading features.

The experiments on bearing life have been conducted through the test samples in order to measure whether the products could meet the manufacturing requirements and also to find the problems of slewing bearing in manufacturing. The mature product of testing machines such as ABLT, ZS series are not widely used and there are no unified national standards or industry standards for fatigue testing machine [1]. The fatigue life test has become an effective way to evaluate various index with unpredictable factors which influence the fatigue life due to the imperfect theory of slewing bearing $[2,3]$.

As a virtual instrument development platform, LabVIEW has been widely used in many fields such as aviation, aerospace, communication engineering, automotive, semiconductor and biomedicine. Its graphical nature makes it ideal for test and measurement, automation, instrument control, data acquisition, and data analysis applications. Using the virtual instrument as the platform for rolling bearing fault diagnosis, the virtual instrument technology is applied to fault diagnosis field which can realize the function of signal collection, analysis and feature extraction. It is more flexible and economical than traditional mechanical fault diagnosis instrument $[4,5]$.

\section{Operating Mode Selection}

According to the bearing fatigue life theory, the bearing's life is related to its load. The operating mode would be selected mainly according to the two load capacity curve of slewing bearing: static 
and dynamic load capacity curve. The static load curve refers to the situation when slewing bearing rolling element and raceway contact point reach to allowable stress of the bearing static load carrying capacity. The dynamic load curve refers to dynamic load capacity when a group of same series slewing bearings get to $90 \%$ of the service life at more than one standard working condition. All the load situation should be carefully considered in designing the testing machine.

\section{Equivalent Load}

Dynamic load curve of slewing bearing refers to the carrying capacity of the service life at 30000 RPM (Revolutions per Minute) .The selection methods of slewing bearing are listed as follows:

1) Select bearing model with static load capacity curve under static operating condition.

2) Check working life with dynamic load capacity curve under dynamic operating condition.

The goal in designing the tester is to measure the fatigue life of slewing beating under the specific load, so the selection of load point should also meet the requirement of actual conditions. For convenience, the four-point contact ball slewing bearing (model 01.25.500) as shown in Figure 1 was taken as an example.

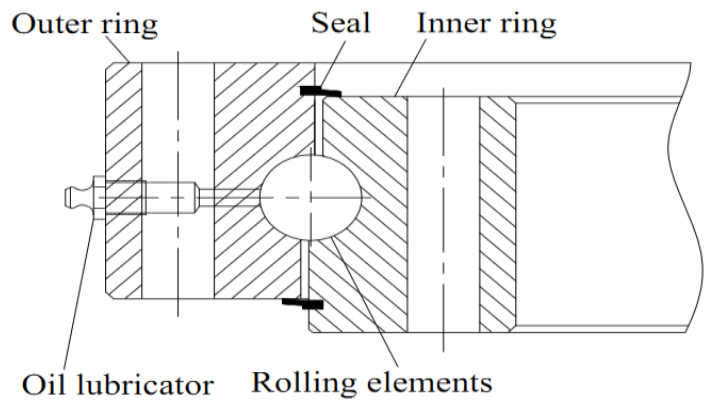

Fig. 1. Single row four-point contact ball slewing bearing

Slewing bearing would be fixed on the bearing support through the mounting holes on the inner and outer ring by the high strength bolt . According to the static condition of the slewing bearing ,the maximum load is noted as the design load point in the load curve. Intersection point of static equivalent load of the total axial force and overturning moment should fall below the capacity curve in Figure 2. Finally, the maximum load of the slewing bearing is the choice of bolt load to proof whether the load points fall below the bolt load curve.

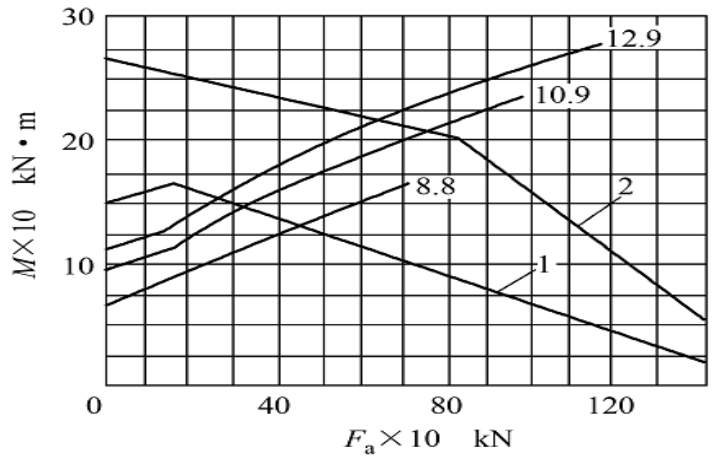

Fig. 2. capacity curve of slewing bearing and bolt

Rated load value:

$$
F_{a}=40 \times 10^{4} \mathrm{~N}, \quad M=12 \times 10^{4} \mathrm{~N} \cdot \mathrm{m}
$$

The equivalent load calculation methods are listed as follows:

Contact angle $\alpha=45^{\circ}$

$$
F_{a}^{\prime}=\left(1.225 F_{a}+2.676 F_{r}\right) f_{s}
$$




$$
M^{\prime}=1.225 M \cdot f_{s}
$$

Contact angle $\alpha=60^{\circ}$

$$
\begin{gathered}
F_{a}^{\prime}=\left(F_{a}+5.046 F_{r}\right) f_{s} \\
M^{\prime}=M \cdot f_{s}
\end{gathered}
$$

Where $F_{a}$ ' is the equivalent axial force, $M^{\prime}$ is equivalent overturning moment, $f_{s}$ is safety coefficient under static condition, $F_{a}$ is total axial load, $F_{r}$ is total radial load, $M$ is overall overturning moment.

Equivalent static load point should fall below the static load curve while the model selection of slewing bearing. There are two kinds of method under the condition of contact angle at $\alpha=45^{\circ}$ and $\alpha=60^{\circ}$ to calculate as shown in Table 1 . Only if the load points match with the curve above, the selected load could meet the strength requirements of the bearing structure. In general it can be used as a load point in designing the testing machine.

Table 1. Load point.

\begin{tabular}{|l|c|c|c|}
\hline & $\begin{array}{c}\text { Static load checking } \\
\text { point } 45^{\circ}\end{array}$ & $\begin{array}{c}\text { Static load checking } \\
\text { point } 60^{\circ}\end{array}$ & $\begin{array}{c}\text { Bolt load checking } \\
\text { point }\end{array}$ \\
\hline $\mathrm{Fa} / 10^{4} \mathrm{~N}$ & 53.9 & 44 & 40 \\
\hline $\mathrm{M} / 10^{4} \mathrm{~N} \cdot \mathrm{m}$ & 16.2 & 13.2 & 12 \\
\hline
\end{tabular}

\section{Extraction of the Vibration Data}

Due to the continuously contact impact between the bearing raceway surface and roller surface, the raceway surface and rolling surface would appear fatigue denudation during rotation of the bearing. The total number of rotation before this kind of rolling fatigue damage is called the fatigue life of bearing.

When there are damages on the surface of bearing, the impact comes out in the process of collision between the rollers and other component part. The band of impact frequency including inherent frequency of the bearing components, sensors, and even other resonator is so wide that it lead to high frequency vibration of the whole system.

Shock pulse caused by bearing fault was pick up by the sensors and enhanced by the circuit resonance. The amplified signal and fault impact can be applied in the spectrum analysis [6]. The sensitivity of fault detection can be greatly improved by the application of sensor and the resonant circuit. On this basis, the theory can be used for rolling bearing fault diagnosis.

\section{Halt Threshold}

The selection of halt threshold and the value of the threshold are important points of accelerated life tests. Margin, kurtosis, peak factor, waveform factor and root mean square are often applied in rolling bearing life test.

The kurtosis value is more sensitive to early bearing fault. The advantage of its high sensitivity becomes limitation at the same time because any disturbance in experiment can cause considerable changes of kurtosis value [7]. Therefore kurtosis value could be too sensitive to be a threshold. Waveform factor can't describe the regular status of bearings accurately while the bearing vibration signal is nonlinear and non-stationary signal .At present, most of the automatic bearing testers are using root mean square value as halt threshold, the root mean square value which reflects the relatively bearing vibration statistics can well predict the health status of bearing fault. The characteristic parameter method is too sensitive to early fault, so further analysis in spectrum is necessary in testing the bearing fatigue life.

\section{Fault Frequency}

Fault frequency needs to be calculated in spectrum analysis. As a result of the type and model of slewing bearing, typical fault characteristic frequencies are totally different. The mathematical 
models for fault characteristic frequency of different bearing element are shown as follows through research of geometry and kinematics of roller bearing.

The frequency of the outer ring:

$$
f_{o}=\frac{1}{2}\left(1-\frac{d}{D_{m}} \cos \alpha\right) f_{r} Z
$$

The frequency of the inner ring:

$$
f_{i}=\frac{1}{2}\left(1+\frac{d}{D_{m}} \cos \alpha\right) f_{r} Z
$$

The frequency of the rolling elements:

$$
f_{\mathrm{RS}}=\frac{1}{2}\left(1-\frac{d^{2}}{D_{m}^{2}} \cos ^{2} \alpha\right) f_{r} \frac{D_{m}}{d}
$$

Where $D_{m}$ is the bearing diameter, $D$ is the average diameter of roller elements, $\alpha$ is the contact angle between the rolling element and inner or outer raceway, $Z$ is the number of rolling elements and $F_{r}$ is the rotational frequency.

Table 2. Slewing bearing fault frequencies

\begin{tabular}{|c|c|c|c|}
\hline & Inner ring & Outer ring & Rolling element \\
\hline Fault frequency (HZ) & 10.6 & 10.1 & 3.3 \\
\hline
\end{tabular}

The slewing bearing fault frequencies shown in Table 2 are calculated when the bearing rotates at 20 RPM (Revolutions per Minute). Different rotational speeds match with different fault frequencies. The contact angle is $45^{\circ}$, the bearing diameter is $500 \mathrm{~mm}$, the ball diameter is $25 \mathrm{~mm}$ and the number of balls is 62 .

Due to low frequency of rotating frequency, the fault frequency of inner ring in the table is relatively close to the outer ring. Higher precision and resolution are required for data acquisition, therefore it still need comprehensive analysis method to judge the result of the power spectral density in further frequency analysis. Compared the fault frequency with acquired signal which gained from Fourier transform, wavelet analysis and HHT (Hilbert-Huang Transform), it makes HHT an efficient method in analyzing the main region of the fault frequency.

\section{Test Station}

Rolling bearing fatigue life tester named ABLT series designed by Hangzhou bearing test centre can be applied to roller bearing with radial force and axial force [8]. Considering the special force situation of the slewing bearing, a certain number of overturning moment need to be loaded additionally.

\section{Loading System}

A pair of slewing bearings were attached to two end of the middle cylinder by bolts. The testing system was driven by synchronous belt wheel in the middle of the cylinder. Operating condition was realized by changing the speed of the electric motor. The schematic of slewing bearing testing platform including the main drive reducer, the hydraulic load and the bearing is shown in Figure 3 . Actual situation of the slewing bearing load was simulated by different value of the loading strength pushed by hydraulic jack. Considering the normal working condition, axial force and radial force were combined into an equivalent load through equation (1) to (4). 


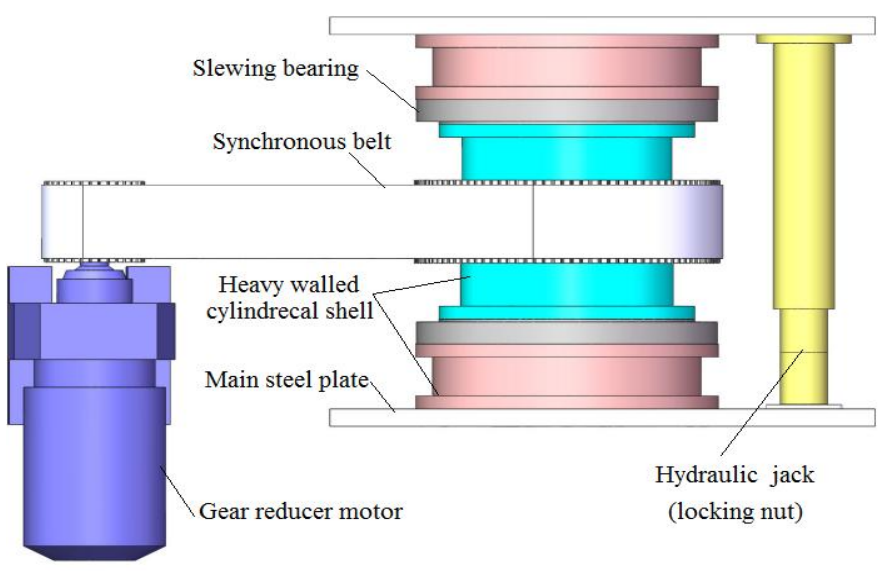

Fig. 3. Schematic diagram of the tester

\section{Measurement and Control System}

Rolling bearing vibration caused by bearing fault spreads out in the form of half spherical wave, so the measure point should get close to the loading area. Due to the different reflection of the vibration signal, measure point should be allocated in three different directions: horizontal, vertical and axial [9]. Taking economy and technique condition into account, radial and axial directions were applied in the test.

\section{Arrangement of Transducers}

The vibration sensor can be divided into three types: displacement sensor, speed sensor and acceleration sensor. Bearing fault signal can be measured effectively by acceleration sensor, so it was installed for the test. The envelope spectrum can intuitively reflect the impact of the vibration. At the same time, the range of the sensor must cover all range of possible fault frequency in order to collect the useful signal.

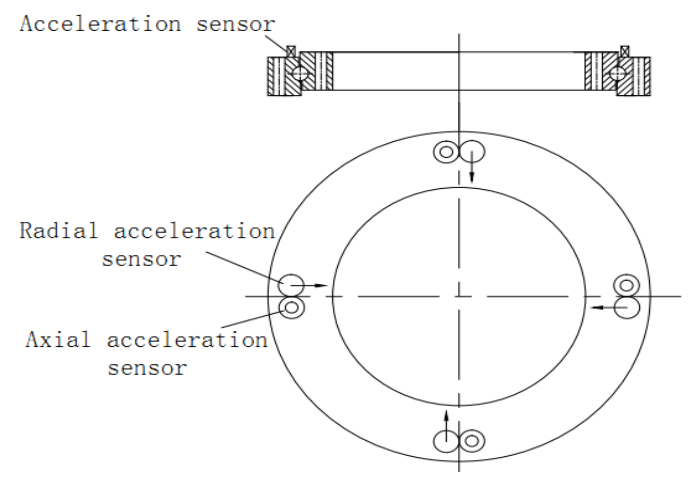

Fig. 4. Allocation of the test points

The bearings were installed on two end of middle cylinder and driven by the synchronous belt wheel. Outer ring was fixed on the base, acceleration sensor was laid out along the outer ring of slewing bearing. The vibration signal of slewing bearing can be easily acquired in this method. Four axial and radial acceleration sensors were arranged intervals at $90^{\circ}$ in the circumferential direction as shown in Figure 4.

\section{Data Acquisition Card}

Data acquisition card (DAQ) is a bridge between external electrical signal and personal computer (PC). It is the core of the acquisition system hardware, including the data collection, A/D conversion and storage modules. Analogy signal was transfer through acquisition channel using different sensors, then the sensor output the signal to PC after pre-treatment the DAQ card analogy channel of transmit 
it to the computer. At the end of the system, the signal collected by DAQ card were analysed by LabVIEW.

The vibration monitoring system in the test machine consists of temperature sensor and accelerator produced by PCB and Advantech. The Advantech PCI-1716 is a 16-bit, 250kS/s high-resolution multifunction card which supports both 16-channel single-ended and 8 differential A/D input, each individual input channel is software-selected. PCI-1716 is a powerful data acquisition card for the PCI bus. It features a unique circuit design and complete functions for data acquisition.

\section{Software Design}

LabVIEW provides a large number of function library and virtual controls in processing signal, like filter function, window function, signal function, time domain and frequency domain function as shown in Figure 5. To meet the law of sampling, high frequency components of the signal must be filtered out by using low pass filter. The signal processing function can be achieved by invoking the function and controls listed above in practical application

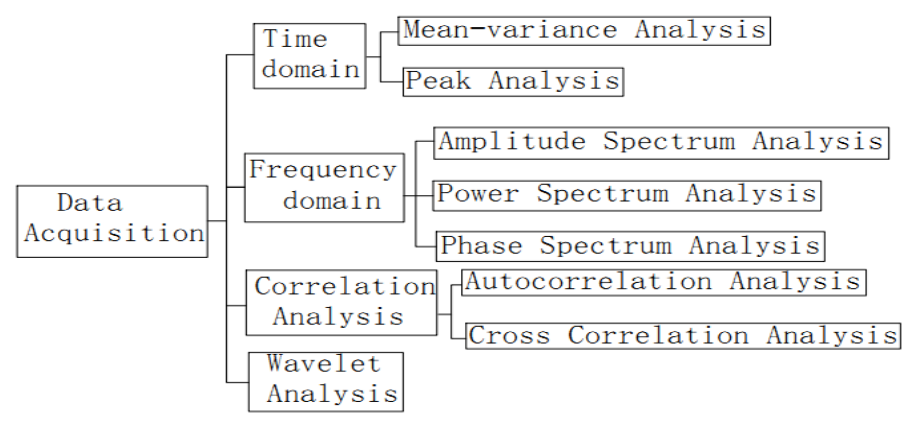

Fig. 5. General structure of data analysis

At first, the vibration intensity of the rotary bearing noise need to be measured and compared with normal signal. We can preliminary estimate system operation in time domain under the exact load condition. If the value exceeds the demand range, further diagnosis need to be taken in frequency domain. The front panel of data acquisition module is shown in Figure 6.

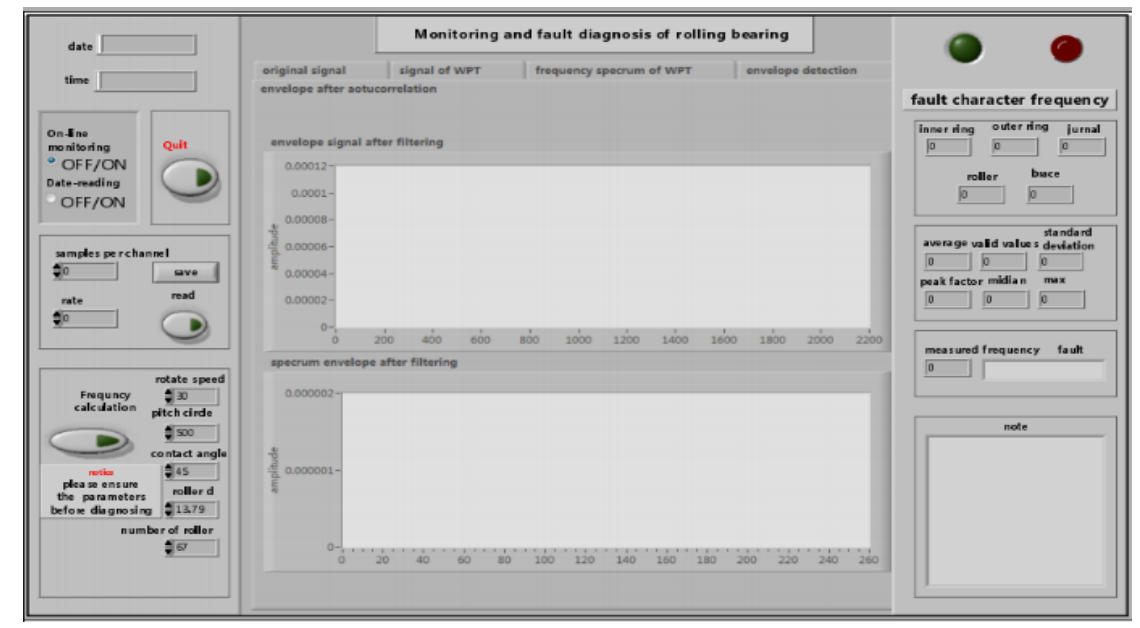

Fig. 6. Front panel of data acquisition module

The analysis is usually carried out with the aid of changing fast Fourier transform (FFT) and Wavelet transform [10]. As shown in Figure 7, the original time domain signals were turned into the envelope detection after a band pass filter module. Envelope detection was constituted of two modules: change module and a rectangular to polar transformation module. Then the signals get through a lowpass filter and reach to spectral analysis. 


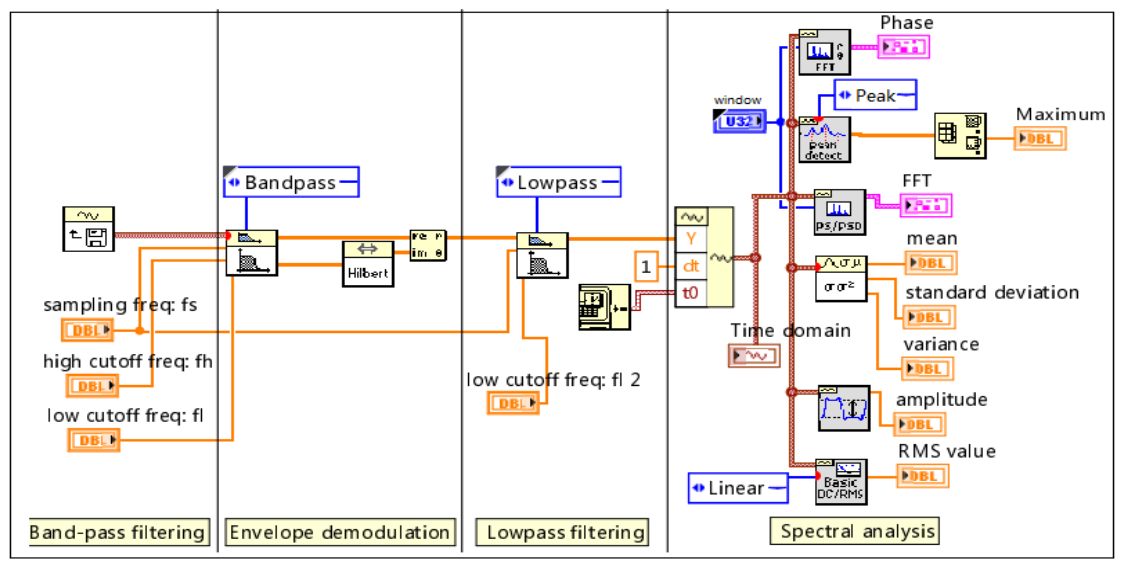

Fig. 7. Resonance demodulation program

In order to find out where the fault occurs in the bearing, the programme need automatic diagnosis tools to find the exact information among the bearing elements like the rolling element and raceway. If fatigue flakes were found by the preceding analysis, vibration signal analysis of the frequency domain would be used to detect the fault. The Fourier spectrum, power spectrum, frequency response function and phase spectrum can be selected in spectrum analysis.

Once the damage of the bearing occurs, the factors of the vibration signal detected by monitoring system would change. When there came the fatigue cracks on the bearing surface, the machine perform the function of halt. Specimens would be removed for further observation and analysing. Bearing fatigue life can be accurately evaluated through the analysis listed above.

\section{Fault Diagnosis}

The steps of the automatic fault diagnosis of the slewing bearing in the technology of resonant demodulations are listed as follows:

1) Input the mode and relevant parameters of the tested slewing bearing.

2) Automatically calculate the bearing fault frequency and generate the fault frequency table.

3) Place the sensors to detect the fault signal.

4) Present digital demodulation and filtering algorithm before spectrum analysis.

5) Compare the fault frequency table with the peak of characteristic spectrum to diagnose the fault position.

6) Diagnose the fault of bearing components and judge degree of damage based on further analysis of the spectrum.

7) The system automatically reports the diagnosis results, gives out halt indication and record the data.

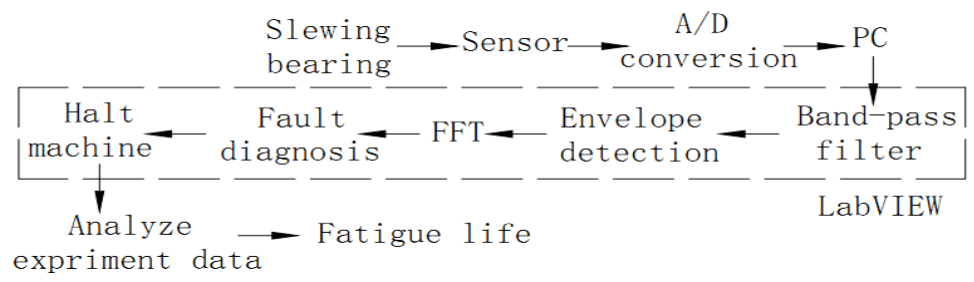

Fig. 8. Flowchart of signal extraction in bearing condition monitoring and prognosis method.

\section{Conclusion}

Fatigue life testing machine is a new type of testing equipment, there are a lot of advantages compared with traditional instrument. According to the resonance demodulation, fault frequency is computed for further analysis. The monitored deterioration index is needed in order to set the threshold when the machine should be stopped or when the predictive maintenance should be carried out. The load 
points of the slewing bearing have been explained. Based on vibration signal acquisition and analysis, the value of bearing fatigue life can be obtained through accelerated life test. As a new instrument of virtual instrument, LabVIEW can define accurate instrument function in data acquisition. The test machine has been in period of adjustment currently. Fatigue Life Testing Machine based on LabVIEW has a great value in fatigue diagnosis and it is the trend of future instrument.

\section{References}

1. X.L. Li, Y.L. Zhang, M.L. Cao, Survey of accelerated bearing life tester and its technique of rolling bearing, Chinese Journal of Ship Research, (2007).

2. H.L. Lou, X.Z Xu, X.L.Li, Study on a life and reliability test evaluation methods of rolling bearings under small sample , Journal of China University of Metrology, 02:124-127, (2011) .

3. D.Xu, Y.C.Xu, X.Chen, Research on Accelerated Life Test for Rolling Element Bearings, Journal of National University of Defense Technology, 06:122-129, (2010) .

4. L.P. Yang, H.T.Li, L. Yang, The LabVIEW program design and application, Electronic Industry Press, (2001).

5. M.Su, Y.Wang, X.G.He, Fault Diagnosis System of Rolling Bearings Based on LabVIEW, Bearing, 09:41-44 , (2010) .

6. Y.T Liu, The Research on the Methods for Condition Monitor and Life Valuation of Low Speed and Heavy Load Rolling Bearings, Wuhan University of Science and Technology, (2006).

7. L. M. Hively, V. Protopopescu, Machine failure forewarning via phase-space dissimilarity measures, Chaos, 14 :408-419, (2005).

8. M.L. Cao, X.C.Liu, X. L. Li, Accelerated Bearing Life Tester (ABLT-6) for Bearings with Outer Ring Rotating, Bearing, 01:50-52, (2009).

9. W.X. Lu, R.S. Du, Engineering Testing \& Signal Processing, Huazhong University of Science and Technology press, (2002).

10. S. Seker, E. Ayaz, Feature extraction related to bearing damage in electric motors by wavelet analysis, Journal of The Franklin Institute, 340 :125-134, (2003). 